\title{
The Office Software Learning and Examination System Design Based on Fragmented Learning Idea
}

\author{
Ling $\mathrm{Xu}^{1}$, Wei $\mathrm{He}^{2}$ \\ ${ }^{1}$ College of Computer Science, Sichuan Normal University, Chengdu, China \\ ${ }^{2}$ College of Physics and Electronic Engineering, Sichuan Normal University, Chengdu, China
}

\begin{abstract}
Fragmented learning is that through the segmentation of learning content or learning time, make learners can use the fragmented time for learning fragmentated content, have the characteristics of time flexibility, learning targeted and high learning efficiency. Based on the fragmented learning ideas, combined with the teaching idea of micro class and interactive teaching, comprehensive utilization of flash animation design software, .NET development platform, VSTO technology, multimedia development technology and so on, design and develop a system integrated with learning, practice and examination of the Office software, which is not only conducive to the effective and personalized learning of students, but also conducive to the understanding the students' situation of teachers, and liberate teachers from the heavy labor of mechanical, focus on promoting the formation of students' knowledge system.

Keyword: fragmented thought, Office software, micro class, operating practice system
\end{abstract}

\section{Fragmented learning}

Fragmented learning is that through the segmentation of learning content or learning time, make learners can use the fragmented time for learning fragmentated content. Because the learning content has been divided, the learning time of each fragment becomes more controllable, which improves the flexibility to learners to control the learning time; learners can also according to their own situation, choose to learning the helpful or intrested learning content, improve the pertinence of learning; due to the learning content is divided, the learning time of a individual pieces of content is short, can protect the learner's learning interest, improve the absorption rate of knowledge, improve the learning efficiency of students.[1][2]

\section{The present situation of Office software teaching}

Computer application ability is the basic skill that everyone should have in the information age. So, freshmen of college will offer computer application basis or computer culture basis courses, help students know the concepts, principles and daily maintenance and grasp the use of the computer. Office software is the main component of this course.

Office software learning has the following characteristics: 1. the main content is operational content, the proportion of concept and theory is small; 2. students' computer application ability are differences due to the information technology learning conditions in middle school and family; 3 . the relevance of each knowledge point is small, which is suitable for the fragmented study; 4. as college students, can not only meet the learning of simple functions, but should master more advanced applications and skills to solve practical problems; 5 . because it is the operation learning content, need to use strengthen means to enhance memory and understanding,

At present, this part of teaching content mainly uses the teaching method: teachers teach and students practice. In practice, this teaching method exposes the following problems: 1 . because of the less of teaching hours, more learning content, students can understand when teacher explanation, but when practice, don't know how to do; 2 . because students' practice mainly in the after-school, often can not get the teacher's guidance in time, 3 . in order to take care of poor students, teachers no time to introduce more valuable advanced application content in the classroom.

About the examination, the current forms are the three as bellow: First, the written examination. In this type of examination, the operation type of content are examed by fill in the blanks, select and simple answer, this examination form is too rigid, and can not really test students operation and application ability on software. Second, free work examination form. Students are required to use the software to create a work to show their ability of learning and application of the software. This form is good at testing of the students' ability of using and innovating of the software, is a good way to qualitative assessment. But is weak of comprehensive examinate the 
grasp of software function of the students, and is not easy to do quantitative evaluation and give points; in addition, this form is need to provide more time to the students to complete their creation work after-class, therefore, the supervision of examination is difficult. Third, sampling work examination form. In this form of examination, the teacher produce a good sample use of the software before examination, requiring students complete the production according to the sample in limited time. The advantage of this form of examination is to facilitate the teacher to quantitative evaluation the situation of students' study of the software function. The disadvantage is that the marking workload is very large.

\section{Learning and examination system design}

Based on the thought of fragmentation, operation strengthening and reducing the unnecessary mechanical repetition of the teacher, we designed the Office software learning and examination system to solve the problems existing in the current teaching and examination. The system is composed of three parts, shown in figure 1 .

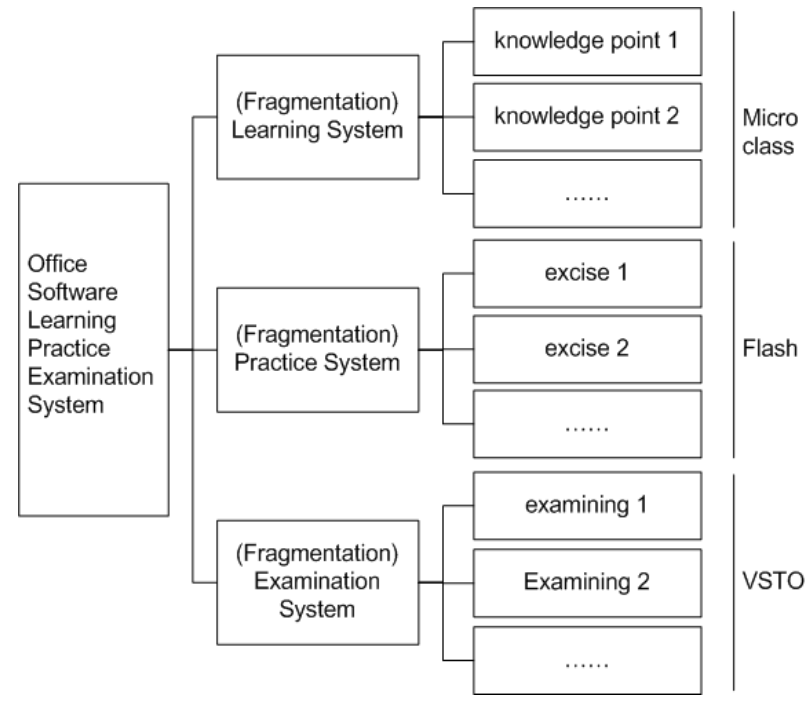

Figure 1 System composition

\subsection{Learning system module}

The learning system module mainly contains the teaching videos, are presented in the form of micro class, to facilitate the students to learn the related concepts, terminology and application operation. The realization of this module first needs to decompose the teaching content to knowledge points. Take the chapter of "the usage of graphic objects" in Word software as an example, the knowledges decomposition and micro classes setting as shown in figure 2 .

Each knowledge point is made into one or more micro class according to the content. According to the characteristics of the content of knowledge point, the different forms of micro lesson can be selected. For example, overview, concept and principle content, can choose the teacher record form; the operation content can choose screen recording mode; principle animation demo

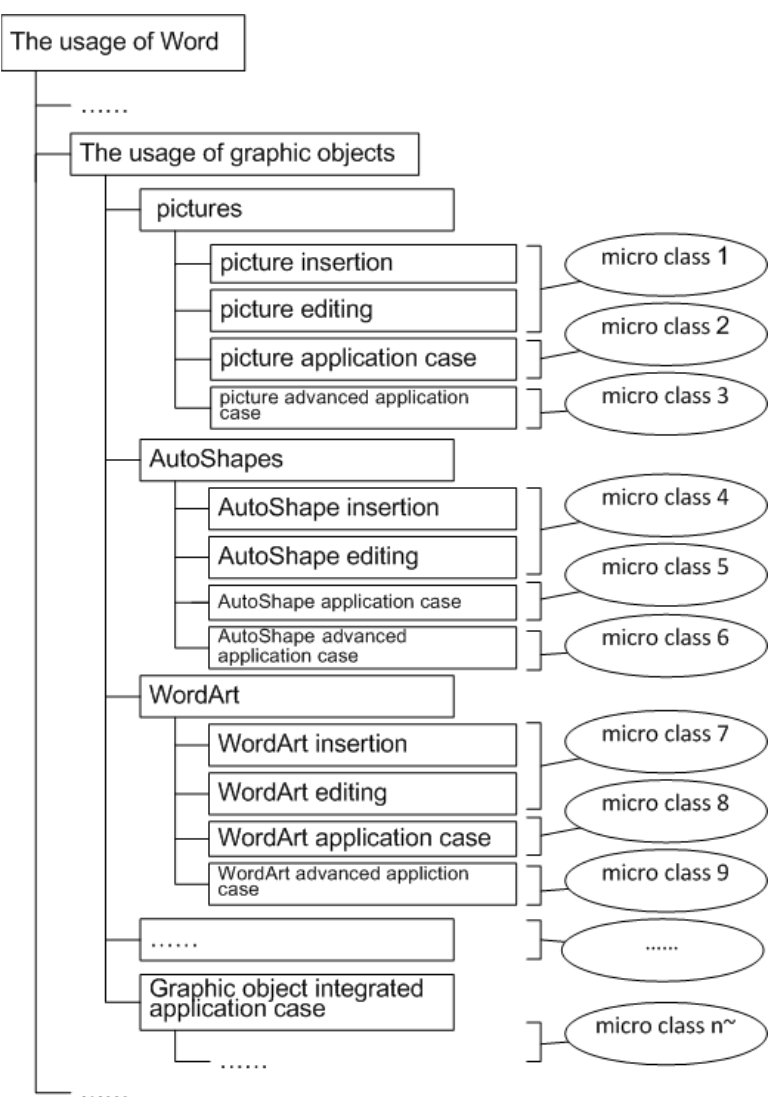

Figure 2 knowledges decomposition and micro classes setting example

content, can be directly translate animation courseware into micro lesson video; and so on. Taking into account the convenience of student learning, the time of micro classes are controled in 5-10 minutes. Finally, organize all the micro class according to the content, all the micro class according to the difficulty is divided into different levels, the first level for students with weak basis to learning the basic content, the second level for students with a certain basis to further study, the third level for students to expand and enhance the advanced application. Clearly identifies each micro class corresponding knowledge points, so, when students learning, they can according to their own situation, quickly choose their own unfamiliar content to focus on learning, so as to reduce the repeated learning time spent on the familiared content. Due to the use of the form of micro class, students can repeat study the content they did not grasp or interested in whenever and wherever possible, thus can get help at any time or place, to avoid the phenomenon of no guidance when encounter problem.

In terms of production, according to the type of the micro lesson, using different production method. For example, for the memoir micro class, in the recording chamber using a camera equipment for recording is a good method; for the demonstration of the operation type of micro class, use screen recording software, record the process of operation and demonstration, and do appropriate post editing, such as increase key annotation, tips emphasize, scene transition, interactive test, etc. finally, in the form of video rendering. This kind of micro class can be produced using Studio Camstia software. 
Camstia Studio software powerful on recording screen and post editing, can record screen action easily in any color mode, including the cursor movement, menu selection, pop-up window, etc. all visible content on the screen; it also can to add markers, increase system icon, add title, add sound effects, increase the effect of mouse etc. in video post-editing. It is a very good tool for making screen recording type of micro classes. For the animation type of micro class, can use animation making software, such as flash or Powerpoint software, generate file to video files format directly after make the animation done.

\subsection{Practice system module}

Practice system module simulate the real software environment to help students carry out the operation exercises, strengthen students' grasp of the operation method and memory, and promote students to quickly and effectively carry out software learning. In the learning process, students often occured such situation: for a given problem, knows what or which function can be used to achieve, but do not know the specific operating methods. The reason is often because the software function is very powerful, students can not find the location of the desired function in so many menu, and don't know how to combined use of multiple functions. So, if we can provide a real software interface, and can be limited to the functional areas of the operation, to help students to quickly grasp the software is very useful. Students' comprehensive application ability of software is built on the independent function of the software. Therefore, the overall realization of this module is still using the idea of fragmentation, according to the decomposition of knowledge points, writing related issues and small tasks to allow students to practice the operation, and give prompt when students encounter problems. The operation of each small problem or task practice idea is: Through screen capture get the real interface of the software - Set the operation areas of the problem (menus, buttons, edit, etc.) to interactive area - Enter the next operation interface if operation right, otherwise, wait for correct operation or get help - Until the last step operation is completed. The implementation process is shown as figure 3 .

Implementation method can be used with the visual programming language (such as $\mathrm{VB}, \mathrm{CH}$, etc.) or interactive multimedia development tools (Authorware, Flash, etc.). Among them, Flash software has the advantages of agility and flexibility, good integration capabilities and so on, so it is better to use Flash to make operation practice of each small problem or small task. [3][4][5]

So, the operation practices of a software are composed of a series of flash file, all flash files are organized according to the classification of knowledge point prepared in advance, and the related information, such as file name, file location, the corresponding knowledge are writed into database, integrate all flash files by VB.NET,

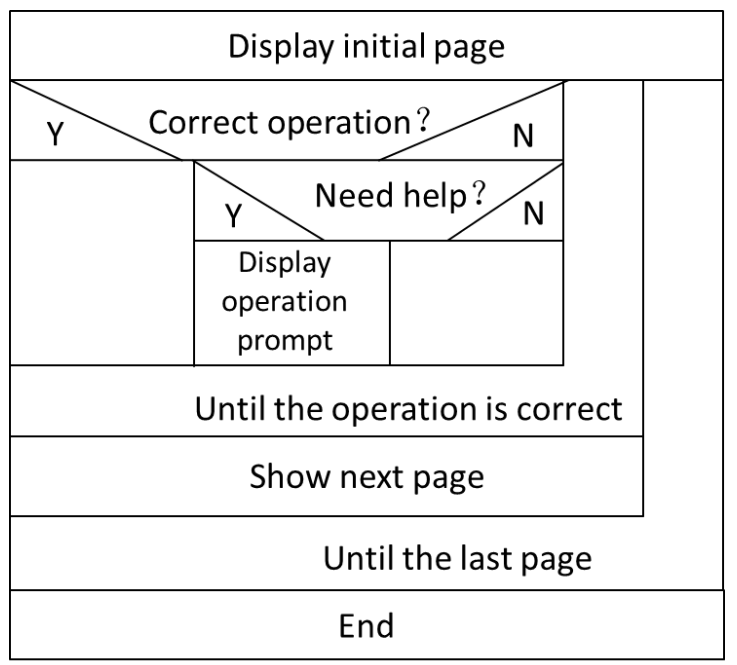

Figure 3. Practice system Implementation process

according to the operator's choice to call and display the corresponding flash file. At the same time, record the situation of seeking help during students doing exercises, in order to do targeted weaknesses training later, to help students to do reinforcement learning on the weak link, so as to quickly master the software usage. Practice system is not only conducive to students quickly become familiar with the software environment, master the operation of software, at the same time, also can make students to get help in the learning process at any time.

\subsection{Examination system module}

The use of software should be in the real software environment, so the final examination should be carried out in a real software environment. In order to fully assess the students' mastery of software function, it is better to use the sample test form, but the shortcoming is the workload of manual marking and the objectivity of the paper. Computer automatic scoring can solve this problem well. Using VSTO technology can realize the automatic reading of Office documents. VSTO's full name is Visual Studio Tools for Office, it added the support of .NET to Word or Excel and other software into the Studio Visual. VSTO convert Word or Excel documents into a .NET class which can be programmed, so that they become control with data binding function, we can encode it like handle the Forms Windows control and other .NET features. VSTO provides a set of Office application object model, use of these object models, we can access the various attributes of the Office document, which can be used to determine the various attributes of the document, to achieve automatic document reading. For example, for the Word document, we can use the Range object to obtain the specific text in the document, read the text format and other information to achieve automatic marking; the use of Document objects, we can get the document level information, to realize the automatic document level marking. The realize framework is shown in figure 4 : 


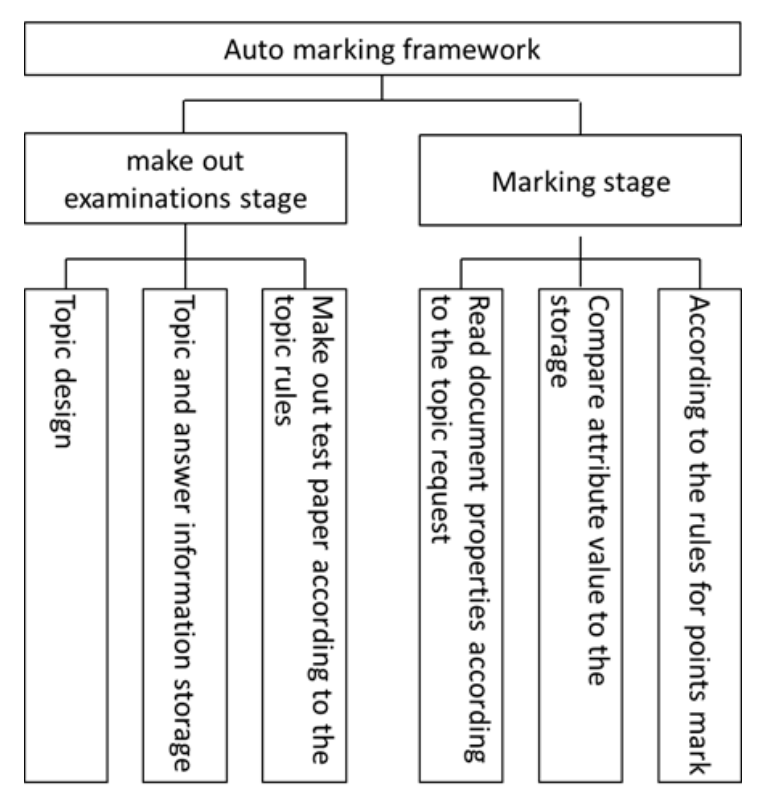

Figure 4. Examination system realize framework

Examination system composed of two parts, exam paper-generating and marking, in the stage of exam paper-generating, the teacher design questions according to the syllabus and the requirements of examination, questions and corresponding answers are written into database, form of item bank. Then, before the examination, select topics from the item band according to the rules of question-making to form the exam paper, be one person one paper. In the marking phase, the system read the students exam information, according to the questions asked, read the related property values of the document, and compare with the answer in library, according to the judgment rules to realize automatic judgment. Examination system ensures comprehensive and randomness of questions through the questionsdesign and paper-make rules, so as to avoid plagiarism phenomenon. At the same time, auto marking function greatly reduces the workload of teacher manual marking and more impersonal.

\section{Conclusion}

Based on the fragmented learning thought, using microclass, solved the problem of basic differences between students and autonomous study in Office software teaching; using simulate actual operating environment but limit operation solve the students' study efficiency in students' autonomous operation practice; use the VSTO technology to achieve the examination papers automatic marking. Such a system is not only conducive to the students' personalized and effective learning, but also for the teacher to master the students' learning situation provides a wealth of data information, and liberate teacher from the mechanical duplication of Labor, can be more energy for teaching itself, should be the future teaching-mode of the software learning. Of course, fragmented learning is not the ultimate goal, the ultimate goal is to achieve the integration and construction of knowledge, to form knowlednage system. Therefore, in the teaching, in addition to help students to learn knowledge more effective with learning system, the teacher should guide students to carry out the processing of knowledge debris integration and migration applications, and promote the formation of students' knowledge system.

\section{References}

1.http://baike.baidu.com/link?url=UzcGD6FQiSrqhHQM G1EDunkFfuQIwiNGW-Yf_GbLVfWEcnfc8FWEw q_idFoSfUj362JHWxY5C4B4tQYQp9d20qBaidu Encyclopedia

2. J.J. Lu, China Medical Education Technology, 10, 508 (2014)

3. W.H.Liu, Development and production technology of micro lesson (Higher Education Press, 2015)

4. P.Z. Gong, Visual Basic.NET programming tutorial (Higher Education Press, 2010)

5. Ch.M. Si, Z.J. Xu, Adobe Flash CS6 courseware production case teaching classic tutorial (Electronic Industry Publishing House, 2015)

6. R. Braunstein, X.M. Tao, Ch. Wang, R.R. Cao, ActionScript 3.0 treasury (Tsinghua University Press, 2012)

7. E. Carter, E. Lippert, Visual Studio Tools for Office 2007: VSTO for Excel, Word, and Outlook (Addison-Wesley Professional, 2009)

8. E. Carter, E. Lippert, VSTO Developer's Guide (Electronic Industry Publishing House, 2008) 